\title{
Anatomy and clinical applications of the mandibular nerve
}

\author{
Krishnaraj Somayaji $\mathrm{S}^{1}$, Rashmi Acharya $\mathrm{S}^{1}$, Mohandas Rao $\mathrm{KG}^{2}$, Venkataramana $\mathrm{V}^{3}$
}

Manipal College of Dental Sciences, Manipal University, Manipal, India. sksomayaji@gmail.com

\begin{abstract}
A thorough anatomical knowledge is very essential for clinical practice and any surgical procedure. Unfortunately anatomical variations can lead to hazards in medical and dental diagnosis and treatment. Such knowledge is very essential even in effective local anesthesia which is an essential part of treatment in patients with many oral disorders. Therefore a normal anatomy and its possible variations are utmost important aspects also in dentistry. One of the structures that dentists very often deal with is the mandibular nerve which therefore needs a thorough review. However, there are not many consolidated literature reviews available regarding its variations and clinical applications. Keeping this in mind, in this article, the authors have brought together available literature on various aspects of mandibular nerve. The final review will be of benefit to clinicians (Fig. 2, Ref. 63). Full Text in PDF www.elis.sk.

Key words: mandibular nerve, inferior alveolar nerve, lingual nerve, collateral innervation, retromolar foramen.
\end{abstract}

A thorough anatomical knowledge is very essential for clinical practice and any surgical procedure. The idea of exact location of various structures in the body prepares the clinician for a better approach in diagnosis and intervention. Unfortunately, the anatomical variations can lead to hazards in medical and dental diagnosis and treatment. Such knowledge is very essential even in effective local anesthesia which is an essential part of treatment in patients with many oral disorders. Therefore a normal anatomy and its possible variations are utmost important aspects also in dentistry. One of the structures that dentists very often deal with is the mandibular nerve which therefore needs a thorough review. However, there are not many consolidated literature reviews available regarding its variations and clinical applications. Keeping this in mind, in this article, the authors have brought together literature available on various aspects of mandibular nerve. The final review will be of benefit to clinicians.

The mandibular nerve, largest division of trigeminal nerve, supplies the mandibular teeth and gums, mucosa of the anterior two thirds of the tongue, floor of the oral cavity, skin of the temporal region, part of the auricle and external acoustic meatus including the tympanum, lower lip, and lower and posterior parts of the face. Developmentally, it is the post-trematic nerve of the first pharyngeal arch and therefore contains the motor fibers to the muscles developed from the first pharyngeal arch. It is a mixed nerve formed by the union of a large sensory root, branch of the lower part of the trigeminal ganglion and a smaller motor root from the pons which courses under the trigeminal ganglion. Both roots

${ }^{1}$ Manipal College of Dental Sciences, Manipal University, Manipal, India,
${ }^{2}$ Melaka Manipal Medical College, Manipal University, Manipal, India,
and ${ }^{3}$ Rajiv Gandhi Institute of Medical Sciences, Adilabad, India

Address for correspondence: Krishnaraj Somayaji S, MDS, Department of Conservative Dentistry and Endodontics, Manipal College of Dental Sciences, Manipal, 576 104, Karnataka, India.

Phone: +919901726764 exit through the foramen ovale and unite just outside the foramen to form the trunk of the mandibular nerve which descends between the tensor veli palatini and lateral pterygoid muscles, gives off a meningeal branch and the nerve to medial pterygoid branch and ends by dividing into a smaller, mostly motor anterior branch and a larger, mostly sensory posterior branch. It contains about 78,000 myelinated fibers. The nerve is about $4 \mathrm{~cm}$ from the surface and a little anterior to the neck of the mandible (1).

The anterior division gives branches to masseter, two deep temporal branches to temporalis muscle, a branch to lateral pterygoid, and a buccal branch which is the only sensory component of the anterior division. The posterior division gives three large branches namely lingual, inferior dental and auriculotemporal (1).

Functional components of mandibular nerve and their intraneural course and connections:

Efferent fibers of the mandibular nerve are derived from the motor nucleus of trigeminal nerve situated in the tegmentum of the upper part of pons. Most of the primary afferent fibers of the nerve are the peripheral processes of the pseudounipolar cells of the trigeminal ganglion. Some of the fibers carrying proprioceptive sensation from the masticatory muscles are the peripheral processes of the cells of the mesencephalic trigeminal nucleus. Electrophysiological observations show that most of the proprioceptive fibers have their cell bodies in the trigeminal ganglion (1).

Primary afferent fibers of the mandibular nerve are of three types. Rapidly conducting myelinated A-Beta fibers respond to light touch and carry proprioceptive sensation. Normally, the activation of such fibers by high-intensity stimulation results in low frequency output in CNS. Usually, these fibers are activated by non-painful mechanical stimulation or pre-pain. Under inflammatory condition, they undergo phenotypic changes to encode painful stimuli $(1,2)$. A-delta fibers are lightly myelinated and have a faster conduction velocity than $\mathrm{C}$ fibers. They transmit sharp and pricking sensation. They primarily respond to noxious mechanical 
431-440

stimuli rather than the chemical or thermal stimuli. Some of the Adelta fibers may be polymodal responding to mechanical, chemical and thermal stimuli $(3,4)$. In the tooth, these fibers traverse the odontoblastic layer and terminate in the dentinal tubules sensitive to mechanical stimulation, and so they respond to stimuli caused by the movement of fluid within dentinal tubules, which leads to sharp pain associated with A-delta fiber activation $(5,6)$. They transmit early shooting pain. C-fibers are unmyelinated slower conducting fibers associated with dull, aching or burning sensations. Most of $\mathrm{C}$ fibers are polymodal, responding to mechanical, thermal and chemical stimuli. In the pulp tissue, C-fibers are more centrally located and sensitized by inflammation (7).

Central processes of primary afferent neurons of the trigeminal ganglion enter the pons as a sensory root of the trigeminal nerve. About $50 \%$ of the fibers divide into ascending and descending branches but others ascend or descend without division. The descending fibers, of which $90 \%$ are less than $4 \mu \mathrm{m}$ in diameter, form the spinal tract of trigeminal nerve which reaches up to the upper cervical segments of spinal cord (1). Spinal nucleus of the trigeminal nerve lies deep to the spinal tract and in the lower medulla, both of them lie deep to the tuberculum cinerium. The spinal trigeminal nucleus corresponds to substantia gelatinosa of the dorsal horn of spinal cord (1). The nucleus descends to the 3 rd or 4 th cervical segmental levels of spinal cord. Fibers of the mandibular nerve lie dorsomedially in the spinal tract of trigeminal nerve.

The arrangement of fibers from different branches of trigeminal nerve within the spinal tract of trigeminal nerve is a subject of controversy. It has long been held that mandibular fibers are dorsal to the fibers of other two divisions and these fibers do not extend much below the mid-olivary level. The results of sections of the spinal tract in trigeminal neuralgia support this view (1). Other workers believe that all the divisions terminate throughout the whole nucleus but ophthalmic division may not project fibers as far caudally as the mandibular nerve (1). Fibers from the posterior part of face terminate in the lower part of nucleus whereas fibers from the anterior part of face terminate at a higher level. This can lead to segmental sensory loss in syringobulbia and is apparently common in tertiary syphilis (1).

Neurons of trigeminal nuclei are second-order or projection neurons and can be divided into three distinct groups on the basis of type of information they receive: 1) low threshold mechanoreceptors, 2) noci-ceptor specific neurons, and () wide-dynamic range neurons. The primary site of termination of nociceptive fibers is the subnucleus caudalis in the caudal part of spinal nucleus which is below, continuous with the dorsal gray column of spinal cord and has been referred to as the medullary dorsal horn (8). It has: 1) central terminals of afferents, 2) interneurons, 3) projection neurons, and 4) descending neurons (9). A-delta and C-fibers terminate primarily in the outer laminae (I and IIa) and in lamina $\mathrm{V}$ within the subnucleus caudalis. Interneurons are composed of inhibitory islet cells and excitatory stalked cells. These interneurons combined, may modulate the nociceptive transmission from primary afferents to projection neurons. Many of the neurons in subnucleus caudalis that respond to cutaneous or tooth pulp stimu- lation are also excited by noxious electrical, mechanical or chemical stimuli derived from jaw or tongue muscles, demonstrating that there is a convergence of superficial and deep afferent inputs via wide-dynamic range neurons or nociceptive specific neurons. The projection neurons that transmit the painful stimuli are of two types: 1) wide-dynamic range neurons receiving input from mechanoreceptors, thermo-receptors and nociceptors, and 2) nociceptive specific neurons which are excited solely by nociceptors. These two types of cells may be responsible for signaling the severity and location of pain, respectively (9). Multiple afferent neurons may form synapses on a single neuron of spinal nucleus (i.e. convergence). This occurs to a much greater degree in deep tissues as opposed to cutaneous tissues. This phenomenon of convergence may result in the clinical finding of pain that radiates beyond the area of tissue injury. When the projection neurons receive inputs from superficial and deep structures, the more superficial inputs usually predominate. Thus the pain originating from deep structures would typically be referred to superficial areas.

The subnucleus caudalis is an important site for relaying the nociceptive input functioning by defined intranuclear projection as part of the pain 'gate Control'. Tooth pulp afferents, wide-dynamic range and nociceptive specific neurons may terminate at higher level of trigeminal nuclei. All rostral trigeminal nuclei project to the subnucleus caudalis (1).

Many of the ascending fibers of mandibular nerve are heavily myelinated and mainly concerned with tactile stimuli, synapse around the small cells in the principle sensory nucleus in the upper level of pons. Other ascending fibers of mandibular nerve enter the mesencephalic nucleus with unipolar neurons whose peripheral processes convey the proprioceptive sensation from masticatory muscles. It is also believed (1) that similar sensations from the teeth as well as from facial and ocular muscles reach this nucleus.

Most of the fibers arising from the trigeminal sensory nuclei cross the midline to ascend as trigeminal lemniscus to reach the ventral posteromedial thalamic nucleus. Some fibers ascend to the ventral posteromedial thalamic nucleus of the same side. Fibers from subnucleus caudalis project to the cerebellum, periaqueductal gray matter, brainstem reticular formation, spinal cord and cranial trigeminal nuclei. The collaterals from the primary and secondary trigeminal afferents reach other cranial nerve nuclei, reticular formation, cerebellum, tectum, subthalamus and hypothalamus (1). Electrophysiological evidence suggests that the mesencephalic nucleus is modulated during masticatory reflexes by its connection with the vagus nerve but the anatomical confirmation of this is lacking (1). The fibers from the facial nerve joining the mandibular nerve are afferents and arise largely from the facial muscles; a minority being propriceptive, the majority being pain fibers. The sensory input from mechanoreceptors in the facial skin, oral mucosa and periodontal membranes to large extent replaces the intramuscular proprioception which is usual in skeletal muscles.

The motor nucleus whose axons constitute the motor fibers of the mandibular nerve contains large and small multipolar neurons in the upper part of pons medial to superior sensory nucleus. It is the branchial efferent nucleus and has small discrete subnuclei whose axons innervate individual muscles (10). The nucleus re- 
ceives the corticonuclear fibers of both sides and from the sensory nuclei. Afferents from the mesencephalic nucleus form the monosynaptic reflex arc for proprioceptive control of the masticatory muscles. It receives fibers from the reticular formation, red nucleus and tectum, as well as medial longitudinal fasciculus and locus coeruleus. These pathways may coordinate the salivary secretion and mastication. Fibers arising from the motor nucleus emerge from the ventral surface of pons forming the motor root of the mandibular nerve which lies anteromedial to the sensory root. The sensory root may be compressed by the superior cerebellar, anterior inferior cerebellar, or pontine branches of basilar artery which may be important in trigeminal neuralgia $(11,12)$.

\section{Course and distribution of branches (Fig. 1)}

Knowledge of the anatomy of mandibular nerve and its distribution is of clinical significance in trigeminal neuralgia in which an agonizingly severe paroxysmal pain radiates over its distribution. Cryoprobe application to the inferior dental nerve may be helpful, as may be chemical neurolysis using alcohol or phenol. Closed percutaneous procedures under radiological control on the trigeminal ganglion via foramen ovale include an injection of chemicals, application of a radiofrequency probe and that of pressure applied by an inflatable balloon. Fractional root section may be performed, i.e.the section of outer fibers may produce analgesia without anesthesia as the fibers responsible for tactile sensation in the medial part are preserved (1).

The meningeal branch from mandibular trunk passes through the foramen spinosum, divides into anterior and posterior branches in the middle cranial fossa to supply the dura mater of the middle and part of the anterior cranial fossa and calvarium. Its posterior branch supplies the mucosa of mastoid air cells. Nervous spinosus contains the postganglionic sympathetic fibers from the middle meningeal plexus.

The nerve to medial pterygoid, the other branch from main trunk, enters the deep surface of medial pterygoid muscle; it gives branches passing through the otic ganglion to supply the tensor tympani and tensor veli palatini muscles (1).

The masseteric nerve, a branch of its anterior division, passes laterally, above the lateral pterygoid on the skull base, anterior to temporomandibular joint, and behind the temporalis tendon, traversing the posterior part of mandibular notch. It supplies the masseter and temporomandibular joint. The course of the nerve is at risk of injury by the anterior dislocation of joint or by excessive protraction of the mandible. There are chances of entrapment of the nerve by the contracting lateral pterygoid. Anterior and posterior deep temporal nerves supplying the temporalis muscle arise from the anterior division. They emerge above the lateral pterygoid and may also be compressed by that muscle. When arising from the buccal nerve, the anterior deep temporal branch can be sandwiched between the upper head of lateral pterygoid and temporalis tendon. The posterior branch may arise in common with masseteric nerve. The nerve to lateral pterygoid is a branch of anterior division normally arising with buccal nerve. The buccal nerve emerges between the two heads of lateral pte- rygoid, passing anterior to the temporalis tendon and appearing on the face deep to the anterior border of masseter to supply the skin over the buccinator, buccal mucous membrane, and posterior part of the buccal gingivae adjacent to the second and third molar teeth (1).

The branches of posterior division of mandibular nerve (lingual, inferior alveolar and auriculotemporal) are of much clinical significance. The lingual nerve (LN) descends between the tensor veli palatini and lateral pterygoid where it is joined by chorda tympani and a communication from the inferior dental nerve. It emerges from the lower border of lateral pterygoid and passes over the medial pterygoid, runs on the medial side of the socket for the mandibular third molar where the nerve can be rolled against the bone just above the posterior end of mylohyoid line of mandible where it is covered only by gingival mucoperiosteum (1). Here it lies usually $2-3 \mathrm{~mm}$ below the alveolar crest and $0.6 \mathrm{~mm}$ from the bone, but in $4.6 \%$ of cases it lies above the alveolar crest (13). Then it leaves the mandible and passes medially and upwards above the styloglossus superficial to hyoglossus (Fig. 1), then between the sublingual gland and genioglossus to enter the tongue. Here it lies deep to submandibular gland and mylohyoid muscles. It winds round the submandibular duct and ascends on the medial side of the duct. It suspends the submandibular ganglion by two roots, and communicates with the hypoglossal nerve (Fig. 1). It supplies the mucosa of the floor of the mouth, lingual gingivae, and the mucosa of the anterior two-thirds of the tongue except for the circumvallate papillae. It carries the pre-ganglionic secretomotor fibers of chorda tympani to the submandibular ganglion as well as the postganglionic parasympathetic fibers from submandibular ganglion to the sublingual and anterior lingual glands. It conveys taste fibers from the anterior two-thirds of the tongue except for the circumvallate papillae which are further carried by chorda tympani (1).

The auriculotemporal nerve arises from the posterior trunk of mandibular nerve by two roots encircling the middle meningeal artery (Fig. 1). One of these roots receives the communicating branch from the otic ganglion which conveys the postganglionic fibers from the ganglion to the nerve to be distributed to the parotid gland. The auriculotemporal nerve then passes back deep to the lateral pterygoid but superficial to the tensor palatini, then between the mandibular neck and sphenomandibular ligament, and then runs laterally behind the temporomendibular joint related to the upper part of parotid gland. It ascends behind the temporomandibular joint and superficial temporal vessels, over the posterior root of zygoma, in front of the auricle to the temporal region where it ends by dividing into superficial temporal branches. Branches of auriculotemporal nerve include: 1) articular branch to temporomandibular joint, 2) glandular branch to parotid gland providing the postganglionic parasympathetic fibers from the otic ganglion to the gland, 3) two branches to external acoustic meatus supplying the skin of posterior part of external acoustic meatus and corresponding outer surface of tympanic membrane, 4) auricular branches to supply the skin over tragus and adjoining upper part of the lateral surface of auricle, and 5) superficial temporal branches supplying the skin of the posterior part of temple (1) (Fig. 1). 


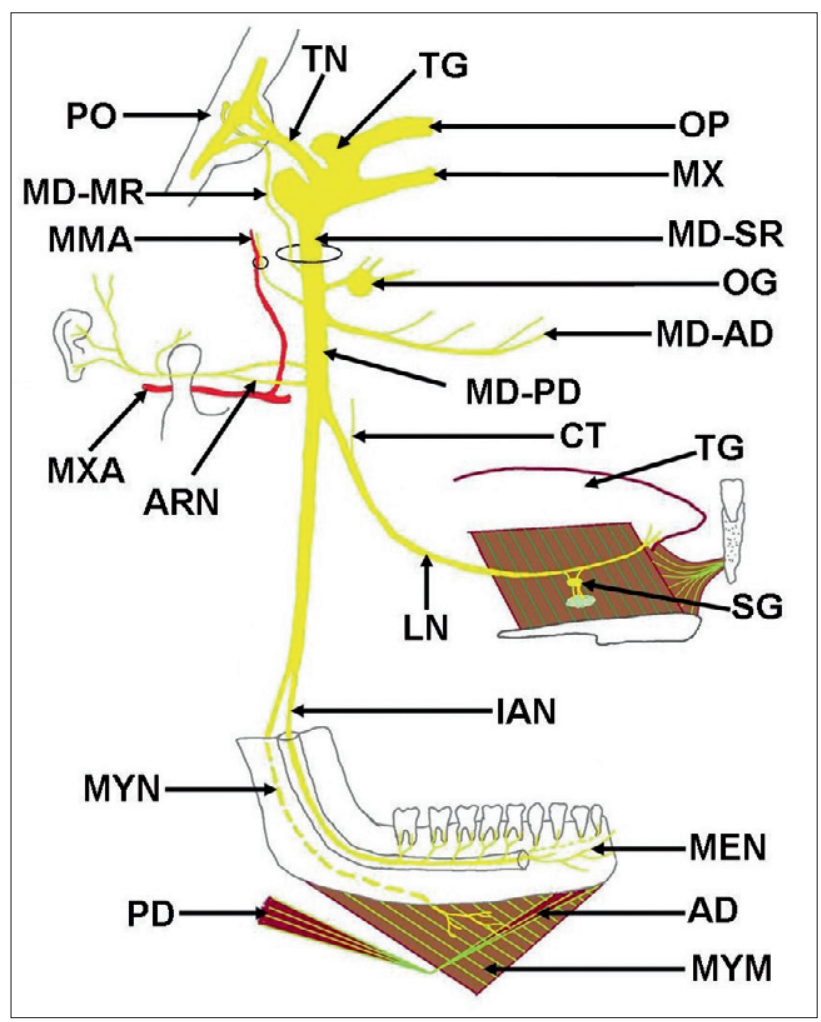

Fig. 1. Figure illustrates the schematic representation of the origin and distribution of the branches of posterior division of mandibular nerve. It can be noticed that the trigeminal nerve (TN) arises from the pons (PO) and reaches the trigeminal ganglion (TG). From the trigeminal ganglion ophthalmic division (OP), maxillary division (MX) and sensory root of the mandibular nerve (MD-SR) arise. The motor root of mandibular nerve (MD-MR) arises separately from the pons (PO) and joins the sensory root (MD-SR) of the main trunk of the nerve. The main trunk of mandibular nerve divides into anterior (MD-AD) and posterior (MD-PD) divisions. From the posterior division of mandibular nerve (MD-PD), inferior alveolar nerve (IAN), lingual nerve (LN), and auriculotemporal nerve (ARN) arise. OG otic ganglion, CT - chorda tympani nerve, TG - tongue, SG - submandibular ganglion, MEN - mental nerve, AD - anterior belly of digastric, PD - posterior belly of digastric, MYM - mylohyoid muscle, MYN - mylohyoid nerve, MXA- maxillary artery, MMA - middle meningeal artery.

The inferior alveolar nerve (IAN) descends deep to the lateral pterygoid, emerges from its lower border and passes between the sphenomandibular ligament and the mandibular ramus to enter the mandibular foramen along with the inferior alveolar vessels. The mandibular foramen is located on the medial surface of the ramus of mandible bordered by the lingula - a lip-like bony prominence that serves a protective function to the nerve and its concurrent artery (Fig. 1). It is in this area that the clinician attempts to deposit the anesthetic solution when performing an inferior alveolar nerve block (also known as mandibular block). The mandible, unlike the maxilla, is composed of dense cortical bone. This is important to the clinician because of its relative inability to pass fluids, thus making infiltration nerve blocks at areas near the apices of the teeth less effective than those administered to corresponding teeth in the maxillary arch. Therefore, the inferior alveolar nerve block is the most popular means of providing sensory anesthesia of lower teeth (14). The mandibular foramen is always situated in the ventral and inferior two-thirds of the ramus without difference in side, sex or age. In spite of the relative variability of the position of mandibular foramen, it is unlikely to be located in the posterior and the superior thirds of the ramus. This area can be considered as a "safety zone" to perform vertical ramus osteotomies of the mandible with statistically low risk of inferior alveolar nerve injury (15)]. The nerve traverses the mandibular canal just below the apices of the teeth and down below the first and second premolars where it terminates by dividing into an incisive and mental branches. In the mandibular canal it gives dental and interdental branches. The dental branches form an inferior dental plexus. From this plexus, the branches enter each tooth through the apical foramina of the $\operatorname{root}(\mathrm{s})$ providing the sensory innervation to the teeth. The interdental branches innervate the alveolar bone, periodontium and gingivae as well as some soft tissue on the ipsilateral side of mandible. The incisive branch continues within the bone, supplying the branches to first premolar, canine and incisor teeth and the associated labial gingivae. The mental nerve passes upwards, backwards and laterally to emerge from the mental foramen between and just below the apices of the premolar teeth. Here it may be visible and is invariably palpable through stretched oral mucosa. It divides into three branches; two of them pass up close to the mucosal surface of the lower lip to supply the mucosa while the third branch supplies the skin of the lower lip and chin (Fig. 1).

Mylohyoid branch arise from the inferior alveolar nerve just before it enters the mandibular foramen (Fig. 1). With its accompanying vessels, it pierces the sphenomandibular ligament, runs along the mylohyoid groove to reach the surface the mylohyoid muscle, and ends by supplying the mylohyoid and anterior belly of digastric muscles. It gives a few filaments to supply the skin over the point of the chin (Fig. 1).

\section{Discussion}

Numerous theories of causation of trigeminal neuralgia have been advanced. These include the presence of a lesion within the trigeminal ganglion or its roots, neoplasms of the head and neck region (e.g. cerebellopontine angle tumor), pressure from blood vessels on the nerve, demyelinization of the trigeminal nerve (e.g. in multiple disseminated sclerosis), and central brain lesions and ischemia of the trigeminal nerve.

In peripheral neurectomy, mostly the mental and infraorbital nerves produce longer duration of pain relief. The major disadvantages are paresthesia and recurrence of symptoms. Some surgeons and neurologists have used bulbar trigeminal tractotomy (cutting of the descending tract of the fifth cranial nerve in the medulla oblongata) to preserve the sensory or proprioceptive function of the nerve. Tactile and dental sensations are preserved in spite of facial analgesia and thermalgesia. On rare occasions, the periapical lesion may act as a trigger point for spontaneous pain similar to that caused by bone cavities after extraction, as proposed by Ratner et al (16). Thus, dental causes must be ruled out as etiologi- 
cal factors when there are trigeminal neuralgia-like symptoms. In such rare cases, unnecessary irreversible treatment such as nerve devitalization or injection therapy, may thus be avoided.

The lingual nerve is at great risk during the extraction of third mandibular molar. About $10 \%$ of patients may have symptoms of nerve damage which are usually temporary. The nerve is also placed at risk in operations to remove the submandibular gland during which the duct must be separated from the lingual nerve (1). Temporary loss of taste is a common side effect of lingual nerve block. The nerve block is easily achieved by slowly depositing anesthetic solution while retracting the needle. Blockage of lingual nerve is an important adjunct to an inferior alveolar nerve block because of the possibility of accessory nerve supply to teeth from the fibres of the ipsilateral lingual nerve (14).

\section{Variations of the mandibular nerve and associated compli- cations}

Effective local anesthesia is an essential part of treatment in patients with many oral disorders. In general practice, this anesthesia is often achieved by injecting local anesthetic solution into the tissue surrounding the nerve innervating the area to be treated. Roughly 4 million local injections are administered by dentists each year. In many patients, effective anesthesia is not achieved. In many cases, this failure is due to anatomical anomalies/variations (14). The clinician must be able to assess whether it is the operator's error or anatomical anomaly is to be blamed for inadequate pain management. Hence, thorough knowledge of normal and probable abnormal anatomy of the jaw with particular emphasis on the nervous system, is necessary to adequately discuss problems arising from anatomical anomalies.

Different variations in the course of the inferior alveolar neurovascular bundle are described (17). Nortje et al, have divided the variations of mandibular canal into four categories: 1) high mandibular canals (within $2 \mathrm{~mm}$ of apices of the first and second molars), 2) intermediate mandibular canals, 3) low mandibular canals, and 4) other variations including duplications or divisions of the canal, apparent partial or complete absence of the canal or lack of symmetry (18). Of the 3,612 subjects studied by them, 47 $\%$ of the canals were high, $49 \%$ were low and only $3 \%$ could not be fitted into the high or low canal categories. The main conclusion of this study was that the mandibular canals are usually, but not invariably, bilaterally symmetrical, and the majority of hemimandibles contain only one major canal. Multiple mandibular canals of the bifid variety characterized by a single mandibular foramen and two nearly equal canals are unusual (18).

Jung et al., found that buccal infiltration with $4 \%$ articaine was as effective as inferior alveolar nerve block in anesthetizing the pulp of the mandibular first molars (19). Kim et al observed 32 Korean adult cadavers and found that there were four communication patterns between the mandibular nerve branches in 12 cases (37.5\%) (20). They suggested that some of these connections were a possible cause of incomplete anesthesia during dental practice.

Chavez et al suggested that during embryonic development there might be three inferior dental nerves innervating three groups of mandibular teeth (21). The canal to the incisors appeared first followed by the canal to the primary molars and subsequently canal to the permanent molars. These canals are directed from the lingual surface of mandibular ramus towards different teeth groups. During rapid prenatal growth and remodeling in the ramus region there is coalescence of canal entrances that are obvious at birth. These observations are consistent with panoramic radiographic findings in which there are different branches supplying the developing tooth buds of permanent third molars and extending into the mandible. This theory also explains the occurrence of trifid mandibular canals in some patients secondary to incomplete fusion of these three nerves. It is reported that inferior alveolar nerve is a single canal in $60 \%$ of cases while in other specimens, the canal was less defined and the nerves and vessels were spread out to occupy a space within the bone rather than a tunnel. As different nerve branches supply different teeth groups, congenital absence of some teeth can be attributed to the lack of development of different nerve branches.

The contribution of parasympathetic fibers from LN to the IAN is also likely; this innervation may participate in the mechanism of secretion of the lower labial salivary glands and may explain the presence of secretion in the absence of efferent input from the otic ganglion to IAN. This communication also suggests the passage of IAN-sensitive fibers; this situation has been reported for other nerves and considered clinically relevant for supplementary innervation of lower molars (22).

Anil et al. reported two cases with variations of IAN, where the maxillary artery passed through a connecting nerve loop, originated in the auriculotemporal nerve and IAN on both sides (23). Complications resulting from intravascular puncture of the maxillary artery due to the administration of local anesthetic can cause a hematoma in this region (the roof of the infratemporal fossa) which can exert soft pressure on other anatomical structures around the artery in this space, such as LN and IAN, generating sensory alterations, which must be considered in the differential diagnosis of facial pain, hyperalgesia and allodynia.

Despite adhering to routine procedures, the failures in surgical interventions and applications of an anesthetic agent may be due to nerve communication that occasionally exists between the branches of posterior division of the mandibular nerve. In the presented variation, not only the area supplied by $\mathrm{LN}$ will be affected but also the mobility of the three nerves will be markedly restricted due to their distal communication while the symptoms may aggravate during the contraction of pterygoid muscles.

Afsar and colleagues suggested that dentists consider the use of panoramic radiographs in locating the mandibular foramen rather than relying on bony landmarks (24). Lacouture and colleagues found that the proximal portion of the maxillary artery crossed the posterior ramus of the mandible closer to the level of mandibular foramen than has been taught traditionally (25). This same study showed a significant incidence of inferiorly directed looping of the maxillary artery immediately above the level of the mandibular foramen (25). To prevent arterial complications in the event that the traditional approach to the foramen fails, the clinician should avoid moving the needle higher along the 
$431-440$

medial ramus than it was placed on original insertion to prevent significant hemorrhage.

The inferior alveolar nerve can arise high in the infratemporal fossa and travel to the base of coronoid process (above and anterior to the mandibular foramen) to enter the mandible (26). These branches carry sensory innervation to the second and third molars. Branches of the mandibular division or of its inferior alveolar or buccal branch may enter the mandible also in the retromolar fossa area and carry sensory fibers to the first and third molars (26).

As described by Nortje, IAN may lie a few millimeters below the roots of teeth or take a course much lower in the mandible near its lower border (27). More commonly the nerve lies near the buccal side of mandible (28). It may be intimately related to the third molar tooth and may groove its root. Cortical defect size on a maxillofacial CT has a high sensitivity and specificity for predicting intraoperative IAN exposure during third molar removal (29). The loss of inferior alveolar canal cortical integrity is associated with an increased risk of experiencing paresthesia after mandibular third molar extraction (30). Boffano and Gallesio have reported a case of a patient who did not show either paresthesia or anesthesia after the surgical removal of a mandibular residual cyst that exhibited adherence to the inferior alveolar nerve bundle (31). One of the main risk factors of developing permanent sensory dysfunction in the distribution of IAN is related to the surgical skills/experience of the operator. Other factors are associated with the type of impaction, and radiographic proximity of the tooth to the inferior alveolar nerve. Such long-term complications can affect the patient's quality of life (32). Khan et al, have observed three cases of maxillary artery passing through the inferior alveolar nerve, splitting it into superficial and deep divisions out of 50 infratemporal fossae dissected by them. Such entrapment of the maxillary artery may cause numbness or headache and may even interfere with injection of local anaesthetics into the infratemporal fossa (33). Similarly, ocular complications, transient loss of vision, diplopia, and blanching of the skin of the infraorbital region were reported in a female patient after an inferior alveolar nerve block for extraction of the permanent mandibular left third molar tooth. Injection of anesthetic solution into the maxillary artery could result to such complications (34). Ortu and Moriggl noted that the maxillary artery passed between the inferior alveolar nerve and the lingual nerve (35). There was also a connection between these two nerves just behind the maxillary artery. The artery passed medial to the inferior alveolar nerve and lateral to the lingual nerve. They also found that the maxillary artery passed lateral to the inferior alveolar and lingual nerves on both sides and an anastomotic branch between the auriculotemporal and the inferior alveolar nerves bounded the artery below. The maxillary artery was compressed by this loop (35).

Clinical studies in endodontics have found a failure with IAN block occurring between $44 \%$ and $81 \%$ of times. Anatomic variation and the fact that the nerves are deeply placed might not allow objective location of the neurovascular bundle. Studies have shown anesthetic success rates of $75-97 \%$ with peripheral nerve stimulator (36). Although Hannan et al (37) found the nerve block administered with ultrasound accurate, it did not result in a more successful pulpal anesthesia. Berns and Sandove found 25\% of accurate blocks using radio-opaque dyes to result in anesthetic failure due to migration of anesthetic solution following the path of least resistance while this was determined by facial planes and structures encountered in the pterygomandibular space (38).

Many dissection studies have revealed that the anatomy of IAN is more complicated than previously thought. In fact, it comes with the alveolar artery into subcanals, forming a sort of plexus. Moreover, the neurovascular bundle was shown to be in contact or close to the lingual cortical plate between the mandibular and mental foramina. A study of adult dried mandibles reported that the upper border of the mandibular canal is located 3.5 to $5.4 \mathrm{~mm}$ below the root apices of the first and second molars. Kilic et al reported a location of the mandibular canal at a mean distance of $10.52 \mathrm{~mm}$ above the inferior margin of mandible. The mean maximum diameters of the mandibular canal, inferior alveolar nerve, inferior alveolar artery, and inferior alveolar vein were 2.52, 1.84, 0.42 , and $0.58 \mathrm{~mm}$, respectively. This study found that the inferior alveolar nerve often gives rise to several branches at each level (range 0-3) (39). There are also reports of concomitant absence of the ipsilateral inferior alveolar canal and mental foramen. The variations in nerve architecture like these are of importance to clinicians who deal with surgery of facial skeleton (40). The results of meta-analysis conducted by Atieh suggest a reasonable diagnostic accuracy for panoramic radiography in the preoperative evaluation of the relationship between third molars and the canal (41).

The apices of the third molar are closest to the alveolar nerve. When viewing the anatomic relation between IAN and mandibular third molar root apices using dental 3D-CT, contact of the two anatomic structures results in an increased risk for IAN exposure or injury (42). Damage to IAN is a severe complication after mandibular third molar extraction that occurs in 0.4 to $8.4 \%$ of cases. CT scan has become a more popular modality for preoperative evaluation of mandibular third molars that provide three-dimensional information about IAN to the third molar tooth. Cone-beam CT (CBCT) produces superior images compared with panoramic radiography and thus it is a better tool for prediction of IAN injury. A cortical interruption of the inferior alveolar canal as viewed on CT imaging may mean a direct contact between IAN and mandibular third molar. It may indicate a higher risk for experiencing parasthesia after mandibular third molar extraction than that when the cortex is intact (43). Inferior alveolar nerve may be damaged during the extraction of the impacted lower third molar tooth. The roots of such teeth are commonly grooved and very rarely perforated by the nerve. It may be damaged in fractures of the posterior toothbearing part of the mandible. It is also reported that the apex of the second molar is $3.7 \mathrm{~mm}$ from the upper border of the mandibular canal and that the mesial root apex of the first molars are farther away from the alveolar canal by about $6.9 \mathrm{~mm}$. Thus, although the relationships between IAN and molar root apices vary, these structures are sometimes very close, allowing the pathologic periapical conditions to affect the nerve structures in the mandibular canal.

IAN can be damaged by traumatic-compressive or toxic injuries, usually resulting in neurapraxia of this nerve. In fact, whenever the causative agent is removed, the damage of the Schwann cells and the impairment of the myelin sheath can heal completely, 
thus making the clinical recovery predictable. If the irritant is not removed or the injury causes an anatomic breakdown of the nerve, IAN can undergo axonotmesis or even neurotmesis and the subsequent paresthesia will almost never resolve. IAN lateralization results in substantial degrees of tissue disorganization at the microstructural level because of the presence of edema. However, at the ultrastructural level, small amounts of fiber degeneration are observed (44). In cases of IAN injury, the delivery of human NGFbeta in the gel leads to better acceleration of recovery over the saline delivery. It provides a possible way to enhance the recovery of nerve injuries in craniofacial distraction osteogenesis clinically (45).

\section{Accessory innervation of teeth by mylohyoidid nerve}

Mylohyoid nerve originates from the inferior alveolar nerve before the nerve enters the mandibular foramen. It has been shown to contain afferent fibers from some mandibular teeth and efferent fibres to mylohyoid and anterior belly of the digastric and thus is the cause of many cases of anesthetic failure (46). The incidence of mylohyoid innervations to the mandibular teeth is approximately $60 \%$. It carries sensation from the pre-molar, canine and incisor teeth (25). One of the studies has even reported innervation of the first molar by mylohyoid nerve (47). There are reports of branches of the mylohyoid nerve entering the mandible through the retromental foramina associated with the lingual cortical bone in the vicinity of the second premolar tooth. The mylohyoid nerve arises from the inferior alveolar nerve about 5 to $23 \mathrm{~mm}$ above the level of the mandibular foramen and enters the mandible at a point distant to the mandibular foramen (47). Therefore, the deposition of local anesthetic in the vicinity of the mandibular foramen during administration of an inferior nerve block most often does not block the mylohyoid nerve. Hence, it is usually recommended to perform the mylohyoid nerve block in the vicinity of the retromental foramina.

Wilson and colleagues have observed the branching of mylohyoid nerve at an average of 14.7 millimeters from the entry of the inferior alveolar nerve into the mandibular foramen (48). This distance could be beyond the area of diffusion of the deposited anesthetic in a conventional mandibular block, thus allowing the accessory nervous supply to continue to transmit pain signals. Madeira et al. have demonstrated the contiguity of a supplementary branch of mylohyoid nerve with either the incisor teeth or gingivae or the incisive nerve proper (49). This should lead the dentist to evaluate the possible role of accessory innervations of the mandibular teeth by mylohyoid nerve in patients who report pain during dental surgery after receiving the mandibular block. Although, the technique described by Sillanpaa et al. of injecting anesthetic solution directly into the posterior aspects of the muscle is plausible, extraoral nerve blocks are not widely accepted in general dentistry and should be avoided (50).

\section{Mandibular nerve and Gow-Gates technique}

Gow-Gates technique is a method of nerve block described by the Australian dentist George Gow-Gates. It is used to provide anesthesia to virtually the entire mandibular nerve. During this technique, anesthesia is injected using a 25-Gauge needle just distal to maxillary second molar below the insertion of lateral pterygoid $(51,52)$. This "high mandibular block" permeates nearly the entire pterygomandibular space with the final optimal deposition of the anesthetic solution just inferior to the insertion of external pterygoid muscle where the pterygomandibular space is largest. Because the pterygomandibular space contains limited fascial restricting planes, the anesthetic solution may infiltrate throughout the space to anesthetize the inferior alveolar, lingual and buccal nerves. The branching of mylohyoid nerve often occurs higher than the deposition of solution in the standard inferior alveolar nerve block. Jastak et al hypothesized that with the Grow-Gates technique, the mylohyoid nerve will also get sufficiently anesthetized while it is still in the pterygomandibular space (53). Watson and Gow-Gates reported a $100 \%$ effective rate with this type of injection (54). Other, perhaps more impartial researchers, have reported anywhere from about $20 \%$ to $30 \%$ increase in efficacy when using this technique (55). Although the incidence of positive blood aspiration is reported to be lower with the Gow-Gates block than the conventional inferior alveolar nerve blocks, the aspiration techniques are imperative when injections are administered so close to major intracranial/extracranial blood vessels. However, there are cases of ophthalmologic emergencies reported during this procedure (56).

\section{Bifid mandibular nerve}

The bifid mandibular nerve is another anatomical variation often observed. This phenomenon can be often easily detected on panoramic radiographs because of the necessary presence of an accessory mandibular foramen and lingulae. Nortje and co-workers used this method of detection in a retrospective study of 3,612 patients (18). They observed the division of mandibular canal in 33 $(0.9 \%)$ cases of which 20 were bilateral and 13 were ipsilateral. These findings suggest that the bifid mandibular nerve could be one of the reasons responsible for inadequate mandibular anesthesia. Considering the number of different patients, Nortje et al. reported that among the 33 patients with bifid canal, no specific pattern of division or duplications was seen in the mandibles studied (18). This makes it difficult to predict the efficacy of inferior alveolar nerve block injections. Because the bifurcation of the nerve occurs before entering the mandibular foramen, a normal inferior alveolar nerve block injection may be insufficient to block the stimulus conduction for both branches. A high inferior alveolar nerve block may be effective in anesthetizing the accessory nerve at or above its branch point. If panoramic radiographs are available, the dentist should be aware of this possible anomaly and look for evidence of an accessory mandibular foramen on radiograph.

In another study, only $0.08 \%$ bifurcation of IAN in 5,000 US Army recruits, aged 17 to 26 was found (57). Furthermore, Langlais and co-workers evaluated routine panoramic radiographs of 6,000 patients, and they found $57(0.95 \%)$ cases of bifid inferior mandibular canals, 19 in males and 38 in females (58). As a second neurovascular bundle may be contained within the bifid ca- 
$431-440$

nals, complications such as traumatic neuroma, paraesthesia, and bleeding could arise because of failure to recognize the presence of this anomaly and its implications. Auluck et al. have reported a case of trifid mandibular canal in their panoramic radiographic study (59). It is important for dentisits to identify the presence of bifid or trifid canals to modify the anesthetic techniques to avoid pain and discomfort to patients. Therefore, the identification of such variations in patterns of mandibular nerve canal is of considerable interest to dentists.

\section{Retro-molar foramen}

The presence of a retro-molar foramen often predicts also the presence of accessory innervations to the mandibular molars. This innervation is thought to arise from either the long buccal or early accessory branches of the inferior alveolar nerve (14). Carter and Keen reported that in almost $40 \%$ of human mandibles with retromandibular foramina, the inferior alveolar nerve was contiguous with molar teeth via retromolar foramina (60). Sawyerand Kiely reported retromolar foamina in $7.7 \%$ of their study without any statistically significant sex differences (61). The dentist who experiences difficulty in achieving local anesthesia of the mandibular molar area should consider accessory innervations via retromolar entry. To overcome this problem, either a small amount of local anesthetic be deposited directly into the tissue of retromolar area, or other high pterygoid entry injection can be done. High pterygoid block would affect the inferior alveolar nerve in the pterygoid space before it gives any branches, while depositing anesthetic solution directly into the retromolar area may affect any accessory nerves from the long buccal nerve as they traverse the soft tissue before entering the mandible (14).

\section{Contralateral innervation of anterior teeth (Fig. 2)}

It is widely accepted that the incisive branch of inferior dental nerve is extensively branched and offers anastomoses over the midline to the counter-lateral side, creating a crossover effect in innervations of the anterior teeth (Fig. 2). The mandible and the mandibular nerve are formed by fusion of first bilateral pharyngeal arches approximately during the fifth week of gestation. The mandible is therefore divided at the midline by the symphyseal cartilage until after the first year of life. Theoretically, this makes the possibility of crossover innervation law. Rood attributed the failure to anesthetize the incisors completely to two things: 1) crossover of the incisive branch of inferior alveolar nerve, and 2) possibility of accessory stimulation from the buccal, facial, mylohyoid, and cervical plexus (62). Such anomalies can pose obvious problems for the clinician to achieve efficient local anesthesia. When complete local anesthesia of the anterior teeth is not achieved after administering an ipsilateral mandibular block, steps must be taken to block the sensation due to any potential crossover and/ /or accessory fibers. One option is the use of a counter-lateral incisive nerve block. This procedure is sensitive technique because the injected solution must pass directly through the opening of the mental foramen to block the nerve. The foramen is normally

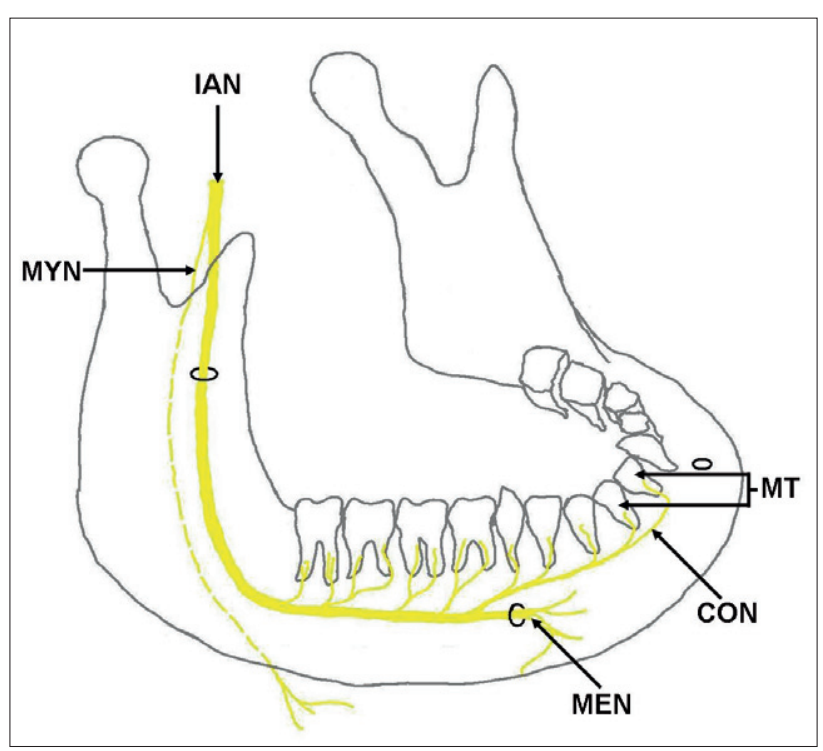

Fig. 2. Figure illustrates the schematic representation of the counterlateral innervation (CON) of mandibular teeth (MT) by the inferior alveolar nerve (IAN). Also shown are mental nerve (MEN) and mylohyoid nerve (MYN).

located in the area of the apex of the second premolar. If possible, the dentist should consult radiographs to help him or her properly place the needle. After the local anesthetic is deposited in this area, finger pressure may facilitate the solution to enter the foramen. Achieving both counter-lateral and ipsilateral nerve blocks would rule out the possibility of failure caused by crossover. Alternatives to bilateral mandibular nerve blocks for procedures on the anterior of mandible include mental/incisive nerve block or periodontal ligament injections. Bilateral mandibular nerve blocks create significant inconvenience for patients because complete mandibular anesthesia leads to postoperative difficulties with speech, eating, drinking and salivary control.

As stated above, the simple supraperiosteal deposition of anesthetic solution to permeate to the apices of mandibular teeth is of relatively low therapeutic value because of the density of an adult cortical plate of mandible. However, if the accessory innervation arises from the branches of the buccal, facial or cervical plexus, and these branches are traveling in the soft tissue of the area previous to the entry into the mandibular foramen, the infiltration blocks, as Rood suggests, may prove successful in deterring the secondary innervations (62). The mandible of the pediatric patient does appear to lack the full bone density, it will acquire in the maturation process, and some success has been shown with mandibular infiltration injections alone within this patient population (14).

\section{Mental nerve parasthesia}

Parasthesia is defined as a sensory disturbance with clinical manifestations such as burning, prickling, tingling, numbness, or itching. Various trigeminal afferent nerves of the mouth can be involved by this alteration. If the mental nerve is affected, the most common complaints include a transient or permanent loss of 
sensitivity of the lip, chin, and oral mucosa which is often associated with a limited intraoral xerostomia. Local and systemic factors have been reported associated with mandibular paresthesias. The local factors are iatrogenic sequelae of endodontic therapy or surgical procedures and the occurrence of acute apical periodontitis or an acute exacerbation of a chronic apical periodontitis. Systemic pathoses include metastatic malignancy, viral infections, multiple sclerosis or Paget's disease. Recently, the possible risk of hypersensitive reactions to potentially toxic or allergenic materials via the root canal has been suggested. Osvaldo reported a case in which mental nerve paresthesia was associated with an adhesive resin restoration placed in direct contact with the pulp of a mandibular second molar (63). The restoration was removed, and the paresthesia had completely resolved after nonsurgical endodontic therapy. Epicutaneous patch testing response indicated a possible causal relationship between the filling material and paresthesia.

\section{Conclusion}

To conclude, it is evident that proper knowledge of normal and abnormal anatomy of mandibular nerve and its branches is essential for the clinicians especially the dentists. Awareness and thorough understanding of available literature on such clinically important structures will always help the clinicians and dental practitioners in handling their patients more safely.

\section{References}

1. Williams PL. Gray's Anatomy: The Anatomical Basis of Medicine and surgery. London: Elsevier, Churchill Liwingstone, 1995; 1230-1239.

2. Neumann S, Doubell TP, Leslie T, Woolf CJ. Inflammatory pain hypersensitivity mediated by phenotypic switch in myelinated primary sensory neurons. Nature 1996; 384: 360-364.

3. Besson J, Chaouch A. Peripheral and spinal mechanisms of nociception. Physiol Rev 1987; 67: 67-86.

4. Lamotte R, Campbell JN. Comparison of responses of warm and nociceptive C-fiber afferents in monkey with human judgments of thermal pain. J Neurophysiol 1978; 41: 509-528.

5. Byers M. Dental sensory receptors. Int Rev Neurobiol 1984; 25: 39-94.

6. Brannstrom M, Johnson G, Nordenvall KJ. Transmission and control of dentinal pain: resin impregnation for the desensitization of pain. J Am Dent Assoc 1979; 99: 612-618.

7. Dubner R, Bennet G. Spinal and trigeminal mechanisms of nociception. Ann Rev Neurosci 1983; 6: 381-418.

8. Dubner R, Hayes R, Hooffman D. Neural and behavioral correlates of pain in the trigeminal system. Pain 1980; 63.

9. Cohen S. Hargreaves KM. Pathways of the pulp, 9th edition, Elsevier Publications, St. Louis, Missouri. 2007; 59-78.

10. Szentagothai J. Functional representation in the motor trigeminal nucleus. J Comp Neurol 1949; 90: 111-120.

11. Jannetta PJ. Arterial compression of the trigeminal nerve at the pons in patients with trigeminal neuralgia. J Neuorosurg 1967; 26:159-162.

12. Klun B, Prestor B. Microvascular relations of thetrigeminal nerve: an anatomical study. Neurosurg 1986; 19: 535-538.
13. Kiesselbach JE, Chamberlain JG. Clinical and anatomical observations on the relationship of the lingual nerve to the mandibular third molar region. J Oral Maxillofac Surg 1984; 42: 565-567.

14. James L, Desantis BS, Charles L. Four common Mandibular nerve anomlies that lead to local anesthesia failures. JADA 1996; 127: 1081-1086.

15. Trost O, Salignon V, Cheynel N, Malka G, Trouilloud P. A simple method to locate mandibular foramen: preliminary radiological study. Surg Radiol Anat 2010. DOI 10.1007/s00276-010-0645-1.

16. Ratner EJ, Person P, Klenman DJ, Shklar G, Socransky SS. Jaw bone cavities and Trigeminal and atypical facial neuralgias. Oral Surg $1979 ; 483-420$.

17. Anderson LC, Kosinski TF, Mentag PJ. A review of the intraosseous course of the nerves of the mandible. J Oral Implantol 1991; 17: 394-403.

18. Nortjé CJ, Farman AG, Grotepass FW. Variations in the normal anatomy of the inferior dental (mandibular) canal: a retrospective study of panoramic radiographs from 3612 routine dental patients. Br J Oral Surg 1977; 15: 55-63.

19. Jung IY, Kim JH, Kim ES, Lee CY, Lee SJ. An Evaluation of Buccal Infiltrations and Inferior Alveolar Nerve Blocks in Pulpal Anesthesia for Mandibular First Molars. J Endod 2008; 34: 11-13.

20. Kim SY, Hu KS, Chung IH, Lee EW, Kim HJ. Topographic anatomy of lingual nerve and variation in communication pattern of the mandibular nerve branches. Surg Radiol Anat 2004; 26: 128-135.

21. Chavez LME, Mansilla LJ, Pompa JA, KajerI. The human mandibular canal arises from threeseparate canalsinnervating different tooth groups. J Dent Res 1996; 75: 1540-1544.

22. Suzao I, Cantin M, Zavando D. Inferior alveolar nerve block anesthesia via retromolar triangle, an alternative for patients with blood discrasias. Oral Surg Oral Med Oral Pathol 2008; 13: 43-47.

23. Anil T, Peker HB, Turgut IN, Gülekon, Liman F. Variations in the anatomy of the inferior alveolar nerve. Br J Oral Maxillofac Surg 2003; 41: 236-239.

24. Afsar A, Haas DA, Rossouw PE, Wood RE. Radiographic localization of mandibular anesthesia landmarks. Oral Surg Oral Med Oral Pathol Oral Radiol Endod 1998; 86: 234-241.

25. Lacouture C, Blanton PL, Hairston LE. The anatomy of the maxillary artery in the infratemporal fossa in relationship to oral injections. Anat Rec 1983; 205:104A.

26. Haveman CW, Tebo HG. Posterior accessory foramina of the human mandible. J Prosthet Dent 1976; 35:462-468.

27. Nortje CT, Farman AG, Grotepass FW. Variations in the normal anatomy of the inferior dental (mandibular) canal: A retrospective study of panoramic radiographs from 3,612 routine dental patients. Br J Oral Surg 1977; 15: 55-63.

28. Miller CS, Nummikosi PV, Barnett DA, Langlais RP. Cross-sectional Tomography. A diagnostic techniquefor determinijg the buccolingual relationship of impacted third molars and inferior alveolar neurovascular bundle. Oral Surg Oral Med Oral Path 1990; 70: 791-797.

29. Susarla SM, Sidhu HK, Avery LL, Dodson TB. Does Computed Tomographic Assessment of Inferior Alveolar Canal Cortical Integrity Predict Nerve Exposure During Third Molar Surgery? J Oral Maxillofac Surg 2010.

30. Park W, Choi JW, Kim JY, Kim BC, Kim HJ, Lee SH. Cortical integrity of the inferior alveolar canal as a predictor of paresthesia after third-molar extraction. J Am Dent Assoc 2010; 141: 271-278. 
$431-440$

31. Boffano P, Gallesio C. Exposed inferior alveolar neurovascular bundle during surgical removal of a residual cyst. J Craniofac Surg 2010; 21: $270-273$.

32. Jerjes W, Upile T, Shah P, Nhembe F, Gudka D, Kafas P, McCarthy E, Abbas S, Patel S, Hamdoon Z, Abiola J, Vourvachis M, Kalkani M, Al-Khawalde M, Leeson R, Banu B, Rob J, El-Maaytah M, Hopper C. Risk factors associated with injury to the inferior alveolar and lingual nerves following third molar surgery-revisited. Oral Surg Oral Med Oral Pathol Oral Radiol Endod 2010; 109: 335-345.

33. Khan MM, Darwish HH, Zaher WA. Perforation of the inferior alveolar nerve by the maxillary artery: An anatomical study. Br J Oral Maxillofac Surg 2009. [Epub ahead of print]

34. Al-Sandook T, Al-Saraj A Ocular complications after inferior alveolar nerve block: a case report. J Calif Dent Assoc 2010; 38: 57-59.

35. Ortu G, Moriggl B. Studies about the topography of the maxillary artery within the infratemporal fossa. Anat Anz 1991; 172: 197-202.

36. Simon F, Reader AL, Melisa D, Nusstin J, Beck M. A prospective, randomized single-blind study of the anesthetic efficacy of the inferior alveolar nerve block administered with a peripheral nerve stimulator. J Enod 2010; 36: 429-433.

37. Hannan L, Reader A, Nist R, Beck M, Meyers WJ. The use of ultrasound for guiding needle placement for inferior alveolar nerve blocks. Oral Surg Oral Med Oral Pathol Oral Radiol Endod 1999; 87: 658-665.

38. Berns JM, Sandove MS. Mandibular block injection: a method of study using an injected radio opaque material. J Am Dent Assoc 1962; 65: 735-745.

39. Kilic C, Kamburoğlu K, Ozen T, Balcioglu HA, Kurt B, Kutoglu T, Ozan H. The position of the mandibular canal and histologic feature of the inferior alveolar nerve. Clin Anat 2010; 23: 34-42.

40. Manikandhan R, Mathew PC, Naveenkumar J, Anantanarayanan P. A rare variation in the course of the inferior alveolar nerve. Int J Oral Maxillofac Surg 2010; 39: 185-187.

41. Atieh MA. Diagnostic accuracy of panoramic radiography in determining relationship between inferior alveolar nerve and mandibular third molar. J Oral Maxillofac Surg 2010; 68: 74-82.

42. Nakayama K, Nonoyama M, Takaki Y, Kagawa T, Yuasa K, Izumi K, Ozeki S, Ikebe T. Assessment of the relationship between impacted mandibular third molars and inferior alveolar nerve with dental 3-dimensional computed tomography. J Oral Maxillofac Surg 2009; 67: 2587-2591.

43. Park W, Choi JW, Kim JY, Kim BC, Kim HJ, Lee SH. Cortical integrity of the inferior alveolar canal as a predictor of paresthesia after $3^{\text {rd }}$ molar extraction. J Am Dent Assoc 2010; 141: 271-278.

44. Yoshimoto M, Watanabe IS, Martins MT, Salles MB, Ten Eyck GR, Coelho PG. Microstructural and ultrastructural assessment of inferior alveolar nerve damage following nerve lateralization and implant placement: an experimental study in rabbits. Int J Oral Maxillofac Implants 2009; 24: 859-865.
45. Wang L, Cao J, Lei DL, Cheng XB, Yang YW, Hou R, Zhao YH, Cui FZ. Effects of nerve growth factor delivery via a gel to inferior alveolar nerve in mandibular distraction osteogenesis. J Craniofac Surg 2009; 20: 2188-2192.

46. Frommer J, Mele FA, Monroe CW. The possible role of the myloid nere in mandibular posterior tooth sensation. JADA 1972; 85: 113-117.

47. [47] Casey DM. Accessory mandibular canals. N Y State Dent J 1978; 44: $232-233$.

48. Wilson S, Johns P, Fuller PM. The inferior alveolar and mylohyoid nerves: An Anatomic study and relation ship to local anesthesia of the anterior mandibular teeth. JADA 1984; 108: 350-240.

49. Madeira MC, Percinoto C, Silva MG. Clinical significance of supplementary innervation of the lower incisor teeth: a dissection study of the mylohyoid nerve. Oral Surg Oral Med Oral Pathol 1978; 46: 608-614.

50. Sillanpaa M, Vuori V, Lehtinen R. The mylohyoid nerve and mandibular anesthesia. Int J Oral maxillofac Surg 1988; 17: 206-207.

51. Gow-Gates GA, Mandibular conduction anesthesia: A new technique using extraoral landmarks. Oral Surg 1973; 36: 321-28.

52. Malamed SF. in Hand book of local anesthesia. Missouri: Mosby, Elsevier Publications, 2004: 237-242.

53. Jastak JT, Yagiela JA, Donaldson D. Local anesthesia of the oral cavity. Philadelphia: Saunders; 1995; 275-285.

54. Watson JE, Gow Gates GA. The clinical evaluation of the Gow-Gates mandibular block technique. NZ Dent J 1976: 72; 220-223.

55. Levy TP. An assessment of the Gow Gates mandibular block for third molar surgery: JADA 1981; 103: 37-41.

56. A Dryden JA. An unusual complication resulting from a Gow-gates mandibular block. Compendium 1993; 14: 94-100.

57. Grover PS Lorton L. Bifid Mandifular nerve as a possible cause of inadequate anesthesia in the manible. J Oral Maxillofac Surg 1983; 41: $177-179$

58. Langlais RP, Broadus R, Glass BJ. Bifid mandibular canals in Radiographs. J Am Dent Assoc 1985: 110: 923-926.

59. Auluck A, Pai KM. Trifid mandibular nerve canal. Dentomaxillofac Radiol 2005: 34: 259.

60. Carter RB, Keen EN. The intra mandibular course of the inferior alveolar nerve. J Anat 1971; 108: 433-440.

61. Sawyer DR, Kiely ML. Retromolar foramen: amandibular variant importantto dentistry. Ann Dent 1991; 50: 16-8.

62. Rood JP. The nerve supply of the mandibular incisor region. Br Dent J 1977; 143: 227-230.

63. Zmener O. Mental nerve parathesia associated with an adhesive resin restoration: A case report. J Endod 2004; 30: 117-119.

Received February 21, 2010. Accepted April 15, 2012. 